\title{
Microperimetry and multimodal retinal imaging, Edoardo Midena (ed). Part of the series "Essentials in Ophthalmology", Series editor Singh AD. (2014) ISBN 978-3642-4029-99 Springer
}

\author{
Shimon Rumelt ${ }^{1,2}$ \\ Received: 11 May 2015 / Accepted: 14 May 2015 / Published online: 21 July 2015 \\ (C) Springer-Verlag Berlin Heidelberg 2015
}

This book addresses a new concept in ophthalmologymicroperimetry. This technique involves the integration of macular clinical images (including fundus color photographs, scanning laser ophthalmoscopy [SLO], and optical coherence tomography [OCT]) with automated perimetry (absolute threshold at different points of the visual field), and may be applicable in all macular and peripapillary disorders. Practically, it allows better correlation between anatomy and function, as the projection and reflection beams are identical in location. It is performed with the use of new devices for this purpose (MP-1 by Nidek in Japan, and Macular Integrity Assessment by CenterVue in Italy). In addition, the book addresses multimodal retinal imaging techniques such as optical coherence tomography, short-wavelength fundus autofluorescence, and different modes of scanning laser ophthalmoscopy. The book, as the author indicates, may serve as an atlas and diagnosis reference text for macular disorders, and may provide a reference source for both retinal experts and researches. The book was largely written by leading experts in the field from Europe and the US, under the editorial skills of Prof. Midena, a leading figure in the study of the retina.

The 196-page book comprises 19 chapters divided into three sections; each addresses either a different aspect of imaging or a different clinical entity. The first six chapters discuss microperimetry as a new assessment technique, OCT/ SLO, and scotopic microperimetry and multimodal retinal

Shimon Rumelt

shimon.rumelt@naharia.health.gov.il

1 Department of Ophthalmology, Western Galilee Medical Center, P.O. Box 21, 22100 Nahariya, Israel

2 Faculty of Medicine, Bar Ilan University, Zefat, Israel imaging. Chapters 7-18 address various retinal disorders, including different types of age-related macular degeneration, retinal pigment epithelium and choroidal grafts, macular telangiectasia type 2 , diabetes and ischemic retinal disorders, myopia, retinal dystrophy, vitreoretinal interface disorders, inflammatory disorders and toxic retinopathy, and glaucoma, with the last chapter dedicated to low vision. Each chapter includes an introduction and detailed reference list. The text is accompanied by numerous high-quality color microperimetry photographs and other relevant imaging photographs relating the different modalities with clinical presentation.

Multimodal retinal assessment techniques are expected to become predominant in the future. At a minimum, a correlation between anatomical integrity (OCT) and functional integrity (visual fields and multifocal electroretinography) for various vitreoretinal disorders could be developed, but a combination with other modalities may be feasible to assess pre- and postoperative retinal status.

This is the first book on microperimetry and multiple retinal imaging techniques, introducing a multi-dimensional approach to the retina and the integration of our knowledge of the macula in health and disease. It is a valuable tool for those who are interested in integrating pathology and functionality, and who want to broaden their understanding of the complexity of the retina. 